\title{
Learner vs. experimenter determination of subsequences in serial memorization
}

\author{
EDWIN MARTIN \\ University of Kansas, Lawrence, Kansas 66045
}

\begin{abstract}
Five subjects each learned 132 word lists of varying lengths, where half of the lists were segmented into rows by the experimenter and half were left unsegmented for the subject to handle as he might elect. Segmented and unsegmented lists yielded similar length-difficulty relations, indicating that the source of list segmentation is not a determinant of learning difficulty.
\end{abstract}

Martin and Noreen (1974) showed that when a learner is faced with learning a supraspan list of words that has no apparent structure or organization, he segments the list into easily identifiable idiosyncratic subsequences that then become the subjective units of learning. Martin, Fleming, Hennrikus, and Erickson (1977) demonstrated that, for a given kind of verbal material, difficulty of serial memorization is determined solely by the length of the list: Learning difficulty did not depend on whether the list was segmented into few large subsequences or into many small subsequences. Experiment 1 in that report involved subjective, learner-determined segmentation of the lists, while Experiments 2 and 3 involved external, experimenter-determined segmentation. The present report addresses the question of which is better, learner or experimenter segmentation.

\section{METHOD}

The subjects were five volunteers from the introductory psychology course at the University of Kansas. Each memorized 132 lists of common four-letter unrelated words. Of the 132 lists, 66 were segmented by the experimenter into one, two, or three rows of three or four words per row, the remaining 66 lists being unsegmented and in a single row, typed across a strip of computer printout paper. As may be calculated from the information above, a given segmented list was $3,4,6,8,9$, or 12 words in total length. There were 11 different lists for each of these lengths. Similarly, there were 11 different unsegmented lists for each of the same list lengths. The 132 lists were randomly generated anew for each of the five subjects.

Note that one-row segmented lists are indistinguishable from the corresponding three- and four-word unsegmented lists. Nevertheless, separate lists were assigned to the segmented and unsegmented conditions.

Each subject was tested individually in four 1-h sessions. The learning procedure was as follows: A piece of paper with a list typed on it was handed to the subject, who then began to study that list in preparation for a perfect recitation. When he thought he was ready to recite the list, he returned the list to the experimenter, who had been measuring the time taken

This research was supported by Grant BNS 75-15770 from the National Science Foundation. The technical assistance of Deborah J. Hennrikus is gratefully acknowledged. Requests for reprints should be sent to Edwin Martin, Department of Psychology, Fraser Hall, University of Kansas, Lawrence, Kansas 66045. en route to mastery. The experimenter noted the number of seconds of study time and the subject delivered his recitation. After a short pause, the next list was presented. The subject was exhorted to use the minimum time necessary for perfect performance and, for the experimenter-segmented lists, to treat the rows as distinct subsequences.

Subjects 1,3 , and 4 , all males, received blocked trials, where all lists within a block of six lists were of either the segmented or the unsegmented variety. At the beginning of each block the subject was advised which kind of list, segmented or unsegmented, would be forthcoming. Subjects 2 and 5, a male and a female, received segmented and unsegmented lists in random order, with no foreknowledge of which type a given list would be.

\section{RESULTS AND DISCUSSION}

Recitations were not always perfect. The number of recitations containing an error was $17(11,6), 8(4,4)$,

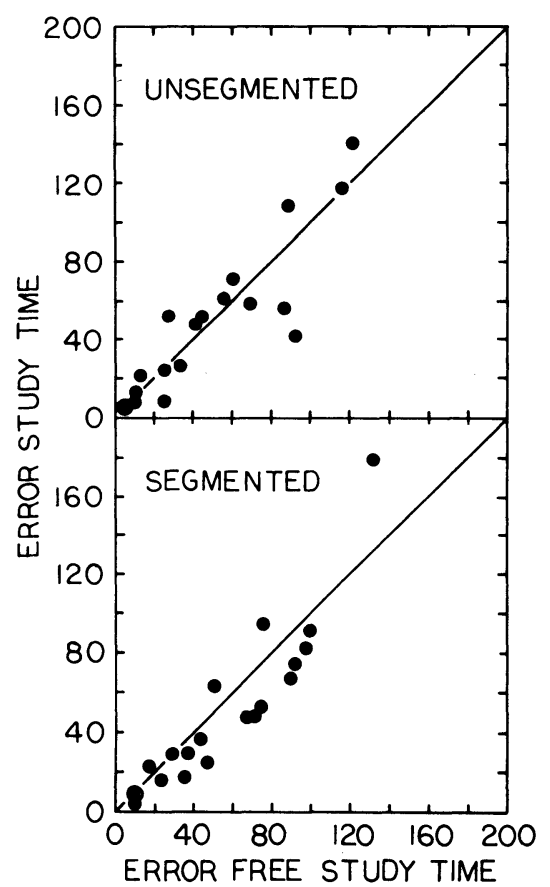

Figure 1. Mean study times (in seconds) for recitations containing an error plotted against those for error-free recitations, for unsegmented and segmented lists. 


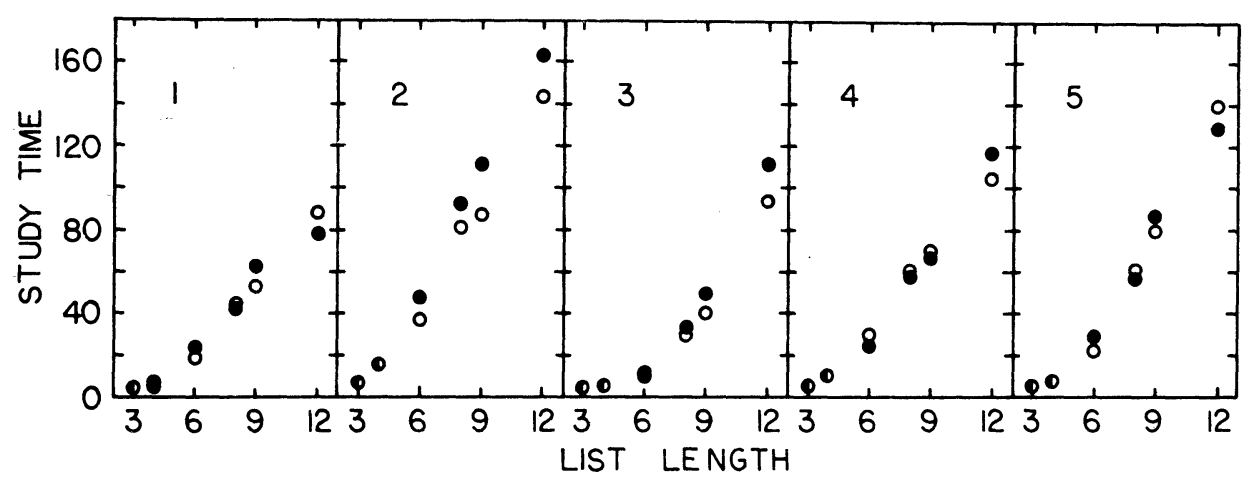

Figure 2. Mean study time (in seconds) as a function of list length for unsegmented (filled circles) and segmented (unfilled circles) lists, for individual subjects.

$14(2,12),-30(13,17)$, and $16(7,9)$ for Subjects 1 , $2,3,4$, and 5 ; in parentheses are the number of recitations that contained an error for unsegmented and segmented lists. Thus, the error rate ranged from $2 / 66=.03$ for unsegmented lists for Subject 3 to $17 / 66=.26$ for segmented lists for Subject 4. As for the unsegmented vs. the segmented contrast, there were, respectively, 37 and 48 recitations containing errors, as may be counted from the parenthetical data above. For combinations of subjects and list lengths for which there were both recitations with and without errors, the geometric mean study time for recitations with errors was plotted against the geometric mean study time for recitations without errors, separately for unsegmented and segmented lists. These plots are shown in Figure 1. Acknowledging that many of the plotted study times for recitations with errors are based on but one or two study times, we note that the points in Figure 1 cluster generally around the diagonal identity function in both panels.

For each list length, a given subject memorized 11 unsegmented and 11 segmented lists. The gecmetric mean study time was determined for each set of 11 lists. These means are plotted in Figure 2, separately by subjects, where filled circles represent unsegmented lists and unfilled circles represent segmented lists. As is apparent, there is no reliable study-time difference between lists that were segmented into discrete rows by the experimenter and those that were left unsegmented for the subject to handle as he might elect. Also, Subjects 2 and 5 , for whom unsegmented and segmented lists turned up on an unpredictable schedule, do not appear to differ in any interesting way from Subjects 1,3 , and 4, for whom unsegmented and segmented lists were blocked into groups of six of a kind.

These results are to be taken as supplementary to those reported by Martin and Noreen (1974) and Martin et al. (1977). Here we have examined the possible role in learning difficulty of who determines the pattern of grouping of the words in a serial list-the experimenter or the subject. We see that for the range of list lengths under study, there is no apparent advantage either for experimenter determination or for subject determination of list structure. There was a tendency toward more recitation errors under experimenter determination, indicating, perhaps, a subjective overestimation of the value of a priori organization, but this tendency seems not to have implicated study times, as may be gleaned from Figures 1 and 2.

\section{REFERENCES}

Martin, E., Fleming, F. G., Hennrikus, D. J., \& ERICKSON, E. A. Studies of the length-difficulty relation in serial memorization. Journal of Verbal Learning and Verbal Behavior, 1977, 16, 535-548.

Martin, E., \& Noreen, D. L. Serial learning: Identification of subjective subsequences. Cognitive Psychology, 1974, 6, 421-435.

(Received for publication April 12, 1978.) 